\title{
Fatores que afetam a comercialização de bezerros em leilões na região Sul do Estado do Rio Grande do Sul, Brasil
}

\author{
[Factors affecting the commercialization of calves in auctions in the southern region of the Rio \\ Grande do Sul State, Brazil]
}

\section{"Artigo Científico/Scientific Article"}

\author{
Pablo Tavares Costa*, Tiago Albandes Fernandes, Rômulo Tavares Costa, \\ Ricardo Zambarda Vaz, Pâmela Peres Farias, Alexsandro Bahr Kröning, Patrícia Pinto Rosa
}

Departamento de Zootecnia, Universidade Federal de Pelotas (UFPel), Capão do Leão-RS, Brasil. *Autor para correspondência/Corresponding author: E-mail: pablocostta@ hotmail.com

\begin{abstract}
Resumo
A rentabilidade dos sistemas de cria bovina é dependente de um conjunto de fatores que atuam em sinergismo. No entanto, ao analisarmos esses elementos isoladamente, o preço de comercialização adquire maior impacto sobre as receitas da atividade. Neste contexto, objetivou-se verificar a influência de características fenotípicas e do período de comercialização (outono e primavera), no preço de bezerros comercializados na região Sul do Rio Grande do Sul. Foram coletados dados de 50 lotes de bezerros, perfazendo um total de 586 animais, previamente e durante o processo de comercialização em leilões realizados no ano de 2017. A uniformidade de estrutura corporal do lote não influenciou o número de lances, a liquidez e o preço final obtido por quilograma dos bezerros $(\mathrm{p}>0,05)$. A uniformidade de padrão racial influenciou o número de lances recebidos pelos lotes de bezerros machos $(\mathrm{p}<0,05)$. Fêmeas foram mais valorizadas no período do outono $(\mathrm{p}<0,05)$. Fêmeas Brangus foram mais valorizadas em relação às Braford e Devon $(p<0,05)$. Fêmeas leves $(\leq 150 \mathrm{~kg})$ receberam maior preço comparadas às mais pesadas $(>180 \mathrm{~kg})$. Machos de pesos intermediários $(150 \mathrm{a} 180 \mathrm{~kg})$ foram mais valorizados que os pesados $(>180 \mathrm{~kg}$ ). O preço praticado pelos machos foi $8,61 \%$ superior ao das fêmeas. Conclui-se que lotes de animais mais uniformes quanto às características raciais recebem maior valorização durante a comercialização. As faixas de peso interferem no preço de venda, sendo os animais mais pesados os menos valorizados. O período de comercialização e a raça dos animais interferem no preço por unidade de peso das fêmeas.
\end{abstract}

Palavras-chave: bovinos de corte; sistema de cria; venda; características fenotípicas.

\begin{abstract}
Profitability of cow-calf systems is dependent on a set of factors that act in synergism. However, when we analyze these elements in isolation, the commercialization price acquires greater impact on the activity revenue. In this context, the aim of this study was to verify the effect of phenotypic characteristics and commercialization period (autumn and spring) on the price of commercialized calves in the southern region of the state of Rio Grande do Sul. Data were collected from 50 calf lots, totaling 586 animals, previously and during the commercialization process in auctions performed in the year 2017. Body structure uniformity within a lot did not influence the number of bids, the liquidity and the final price obtained per kilogram of the calves $(p>0.05)$. Females had a higher price in the autumn period $(p<0.05)$. Brangus females had a higher price than Braford and Devon females $(p<0.05)$. Lightweight females $(\leq 150 \mathrm{~kg})$ had a higher price than the heavier females $(>180 \mathrm{~kg})$. Intermediate weight males $(150$ to $180 \mathrm{~kg})$ had a higher price than heavy males $(>180)$. The price paid for males was $8.61 \%$ higher than that for females. It is concluded that more uniform animal lots in terms of racial characteristics receive higher values during commercialization. The weight ranges affect the sale price, with heavier animals being the least valued. The commercialization period and the animals' breed influence the price per unit of female weight.
\end{abstract}

Keywords: beef cattle; cow-calf system; sale; morphometric characteristics. 


\section{Introdução}

A cria é a etapa menos rentável da pecuária de corte (Oiagen et al., 2009), estando sua lucratividade atrelada aos índices produtivos da propriedade, tais como a taxa de desmame, o peso dos bezerros, os custos de produção (Christofari et al., 2010) e a idade em que as matrizes são expostas à reprodução. Contudo, fundamentalmente, o preço de comercialização dos animais é o fator isolado que pode determinar maiores diferenças na rentabilidade do sistema. Neste contexto, a venda do bezerro adquire papel de grande importância, pois uma má comercialização pode resultar em prejuízo financeiro (Cristofari et al., 2009; Euclides Filho e Euclides, 2010).

No Sul do Brasil, os leilões constituem umas das mais importantes formas de comercialização dos animais (Machado Filho, 1994), onde, se reúnem no mesmo ambiente, compradores e vendedores, sendo os animais expostos em lotes e atribuídos lances sucessivos, em uma espécie de disputa, onde o lance derradeiro permite a aquisição do lote desejado.

O preço de comercialização é definido por meio da interação de diversos fatores, estritamente relacionados à genética e ao fenótipo dos animais, tais como o peso, o sexo, a raça, a presença ou não de chifres, o tamanho e a uniformidade do lote (Schoroeder et al., 1988; Cristofari et al., 2008; Cristofari et al., 2009; Cristofari et al., 2010; Fornari et al., 2016), bem como sofre influência direta do preço praticado com outras categorias bovinas (Sachs e Martins, 2007), em especial as de animais para abate, e, mais recentemente, pelos preços praticados para exportação de bezerros vivos.

O valor da venda dos animais resulta de um processo dinâmico (Machado Filho, 1994), passível de influências externas, não controladas pelo vendedor, que podem resultar em grandes alterações nos diferentes anos de comercialização, bem como nas distintas épocas do ano. Dessa forma, o presente trabalho foi conduzido com objetivo de verificar a influência de características fenotípicas de bezerros e do período de comercialização (outono e primavera), no preço final por unidade de peso vivo de animais comercializados na região Sul do Estado do Rio Grande do Sul.

\section{Material e Métodos}

$\mathrm{O}$ estudo foi baseado na coleta de dados de comercialização de 50 lotes de bezerros, perfazendo um total de 586 animais, provenientes da região Sul do Estado do Rio Grande do Sul (RS), previamente e durante $\mathrm{o}$ processo de comercialização, em leilões realizados durante o ano de 2017 no município de Pinheiro Machado, RS, em duas estações de venda (outono e primavera). Os lotes apresentavam número mínimo de 10 , e máximo de 18 animais, e peso variando entre 130 e $272 \mathrm{~kg}$.

A pesagem dos animais foi efetuada pela empresa responsável pela organização dos leilões, sendo disponibilizado o peso médio de cada lote, em kg. A classificação racial dos lotes foi efetuada por Zootecnista capacitado, avaliando características fenotípicas dos animais e, de acordo com a avaliação do lote, distribuindo-os nas distintas raças, bem como em animais sem raça definida.

A uniformidade de estrutura corporal dos lotes foi estimada previamente ao leilão, observando-se à musculosidade, o tamanho e a estrutura corporal dos animais, mediante metodologia descrita por Koury Filho et al. (2009), e o escore de condição corporal, conforme preconizado por Spitzer (1986). A uniformidade de raça foi avaliada no mesmo momento, conforme características fenotípicas e de pigmentação de pelagem. Para ambas características de uniformidade, foi adotada classificação similar a descrita por Christofari et al. (2009), onde grupos compostos por animais com mais de $90 \%$ de características similares foram classificados como uniformes, quando menos de $50 \%$ dos animais diferiam do restante o lote foi considerado de média uniformidade, e quando mais de $50 \%$ dos animais diferiam, o lote foi tido como desuniforme.

Todos os machos comercializados eram castrados e o lance mínimo inicial para comercialização foi estipulado pela empresa responsável pela comercialização, em relação ao peso vivo médio do lote, sendo de $\mathrm{R} \$ 5,00$ e $\mathrm{R} \$$ 4,50 por quilograma de peso vivo para machos e fêmeas, respectivamente.

Durante o leilão, foram mensurados o tempo de permanência em pista, o número de lances recebidos e os valores iniciais e finais de comercialização de cada lote. Foram calculados a 
valorização, obtida pela diferença entre os preços final e inicial do lote e o preço final por quilograma de peso vivo, conforme metodologia de Christofari et al. (2008).

Os dados coletados foram tabulados em uma planilha do Microsoft Excel®, e uma análise exploratória inicial dos dados foi realizada. Foram determinadas as frequências e as porcentagens de lotes de acordo com cada característica avaliada. Os preços de comericialização (por uniformidade de raça e tamanho, sexo e faixa de peso) formam analisados pelo PROC GLM do SAS (Statistical Analysis System, versão 9.0) e quando verificada significância estatística $(\mathrm{p}<0,05)$, as médias foram contrastadas pelo teste de Tukey. Para analisar os dados de número de lances (por uniformidade de raça e tamanho, sexo e faixa de peso), por serem distribuídos em valores absolutos, usou-se o método não paramétrico de Kruskal-Wallis e, quando verificada significância estatística $(\mathrm{p}<0,05)$, suas médias foram contrastadas pelo teste Dunn efetuadas pelo programa BioEstat 5.3 (Ayres et al., 2007), ao nível de 5\% de significância.

\section{Resultados e Discussão}

A uniformidade de estrutura corporal do lote não influenciou o número de lances, a liquidez e o preço final obtido por quilograma de peso vivo dos bezerros machos e fêmeas (Tabela 1).

Tabela 1. Percentual de uniformidade de lote (PUL), número de lances recebidos (NL) e valor por unidade de peso $(\mathrm{R} \$ / \mathrm{kg})$ de bezerros machos e fêmeas, de acordo com o período de comercialização dos lotes.

\begin{tabular}{|c|c|c|c|c|c|c|}
\hline \multirow[b]{2}{*}{ UEC $^{1}$} & \multicolumn{3}{|c|}{ Machos } & \multicolumn{3}{|c|}{ Fêmeas } \\
\hline & PUL & NL & $\mathbf{R} \$ / \mathrm{kg}$ & PUL & NL & $\mathbf{R} \$ / \mathrm{kg}$ \\
\hline Outono & & & & & & \\
\hline Desuniforme & 19,23 & $3,00 \pm 1,34$ & $5,12 \pm 0,14$ & - & - & - \\
\hline Média Unifor. $^{2}$ & 57,69 & $2,80 \pm 3,10$ & $5,15 \pm 0,24$ & 85,71 & $6,90 \pm 2,09^{\mathrm{A}}$ & $5,05 \pm 0,25^{\mathrm{A}}$ \\
\hline $\begin{array}{l}\text { Uniforme } \\
\text { Primavera }\end{array}$ & 23,08 & $4,10 \pm 2,60$ & $5,24 \pm 0,13$ & 14,29 & $10,00 \pm 1,00^{\mathrm{A}}$ & $5,19 \pm 0,10^{\mathrm{A}}$ \\
\hline Desuniforme & 33,33 & - & - & 12,5 & $5,00 \pm 1,00$ & $4,75 \pm 0,10$ \\
\hline Média Unifor. $^{2}$ & 55,56 & $3,60 \pm 3,30$ & $5,22 \pm 0,24$ & 12,5 & $1,00 \pm 0,00^{\mathrm{B}}$ & $4,49 \pm 0,10^{\mathrm{B}}$ \\
\hline $\begin{array}{l}\text { Uniforme } \\
\text { Média Geral }\end{array}$ & 11,11 & $5,00 \pm 0,00$ & $5,29 \pm 0,10$ & 75 & $2,01 \pm 2,00^{\mathrm{B}}$ & $4,59 \pm 0,17^{\mathrm{B}}$ \\
\hline Desuniforme & 15,62 & $1,87 \pm 2,42$ & $5,12 \pm 0,14$ & 6,25 & $5,00 \pm 1,00$ & $4,75 \pm 0,10$ \\
\hline Média Unifor. $^{2}$ & 62,50 & $3,05 \pm 3,07$ & $5,17 \pm 0,24$ & 50 & $6,10 \pm 3,40$ & $4,84 \pm 0,24$ \\
\hline Uniforme & 21,88 & $4,20 \pm 2,50$ & $5,24 \pm 0,13$ & 43,75 & $3,10 \pm 3,30$ & $4,67 \pm 0,27$ \\
\hline
\end{tabular}

Embora a inexistência de diferenças significativas para o número de lances e preço por unidade de peso, observou-se que, percentualmente, a maioria dos lotes de bezerros e bezerras foi classificada como de média uniformidade ou como uniformes, quanto à estrutura corporal dos animais, sendo poucos lotes identificados como desuniformes. Este fato sugere que os produtores que objetivam a venda estão preocupados com a padronização e apresentação dos animais em pista. Em adição, apenas $6 \%$ do total de lotes ofertados não foram comercializados, sendo todos de machos, classificados como desuniformes, o que pode ressaltar a valia da padronização de estrutura corporal dos animais no momento de formação dos lotes. Embora a não observância de diferenças quanto aos preços praticados para os lotes comercializados, a concentração da totalidade de animais não vendidos na mesma classe, indica que esses tenham sido preteridos em relação às demais classificações.

Ao se analisar os dados de comercialização das distintas classes de estrutura corporal, frente aos distintos períodos (outono e primavera), verificou-se superioridade no número de lances e no valor por quilograma de peso vivo para bezerras vendidas no outono $(p<0,05)$. Não se observaram diferenças nos machos ( $p>0,05)$.

Lotes de bezerros machos classificados como uniformes quanto ao padrão racial receberam maior número de lances quando comparados aos desuniformes $(\mathrm{p}<0,05)$, nos dois períodos do estudo e na média geral, embora não tenham ocorrido diferenças no valor recebido pelos lotes ( $\mathrm{p}=0,0586$, para valores da média geral) em relação à unidade de peso vivo. Nas fêmeas não foram observadas diferenças $(p>0,05)$ para nenhuma das características (Tabela 2).

A maior procura e disputa por lotes compostos por bezerros com uniformidade de 
padrão racial ressalta a importância da seleção de animais dentro das características da raça criada pelo produtor, com o foco na padronização do rebanho e, consequente, maior interesse pelos animais no momento de comercialização. Esses achados corroboram os resultados de Christofari et al. (2008) e Koetz Júnior et al. (2014), de que lotes de padrão racial uniforme têm a preferência dos consumidores e apresentam maior liquidez em relação aos demais.

Tabela 2. Percentual de uniformidade de raça (PUR), número de lances recebidos (NL) e valor por unidade de peso $(\mathrm{R} \$ / \mathrm{kg})$ de bezerros machos e fêmeas, de acordo com o período de comercialização dos lotes.

\begin{tabular}{|c|c|c|c|c|c|c|}
\hline \multirow[b]{2}{*}{$\mathbf{U} \mathbf{R}^{1}$} & \multicolumn{3}{|c|}{ Machos } & \multicolumn{3}{|c|}{ Fêmeas } \\
\hline & PUR & NL & $\mathrm{R} \$ / \mathrm{kg}$ & PUR & NL & $\mathbf{R} \$ / \mathbf{k g}$ \\
\hline Outono & & & & & & \\
\hline Desuniforme & 19,23 & $1,4 \pm 0,9^{b}$ & $5,02 \pm 0,04$ & - & - & - \\
\hline Média Unifor. ${ }^{2}$ & 23,08 & $2,2 \pm 2,4^{\mathrm{ab}}$ & $5,07 \pm 0,13$ & 85,71 & $7,0 \pm 0,0^{\mathrm{A}}$ & $5,03 \pm 0,10^{\mathrm{A}}$ \\
\hline $\begin{array}{l}\text { Uniforme } \\
\text { Primavera }\end{array}$ & 57,69 & $4,4 \pm 2,9^{\mathrm{a}}$ & $5,25 \pm 0,21$ & 14,29 & $6,9 \pm 2,9^{\mathrm{A}}$ & $4,93 \pm 0,25^{\mathrm{A}}$ \\
\hline Desuniforme & 22,22 & $0^{\mathrm{b}}$ & - & 12,5 & $5,0 \pm 0,0$ & $4,75 \pm 0,10$ \\
\hline Média Unifor. ${ }^{2}$ & 66,67 & $2,6 \pm 1,0^{\mathrm{ab}}$ & $5,22 \pm 0,24$ & 12,5 & $1,0 \pm 0,0^{\mathrm{B}}$ & $4,49 \pm 0,10^{\mathrm{B}}$ \\
\hline $\begin{array}{l}\text { Uniforme } \\
\text { Média Geral }\end{array}$ & 11,11 & $5,00 \pm 0,00^{\mathrm{a}}$ & $5,29 \pm 0,10$ & 75 & $2,1 \pm 1,8^{\mathrm{B}}$ & $4,59 \pm 0,17^{\mathrm{B}}$ \\
\hline Desuniforme & 20 & $1,0 \pm 1,0^{\mathrm{b}}$ & $5,02 \pm 0,04$ & 6,25 & $5,0 \pm 2,0$ & $4,75 \pm 0,10$ \\
\hline Média Unifor. $^{2}$ & 34,29 & $2,6 \pm 2,8^{\mathrm{ab}}$ & $5,14 \pm 0,19$ & 12,5 & $4,0 \pm 4,2$ & $4,76 \pm 0,38$ \\
\hline Uniforme & 45,71 & $4,4 \pm 2,9^{a}$ & $5,25 \pm 0,21$ & 81,25 & $4,2 \pm 3,7$ & $4,81 \pm 0,32$ \\
\hline
\end{tabular}

O período de comercialização influenciou a fluidade comercial de fêmeas, frente ao número de lances e ao preço por unidade de peso $(\mathrm{p}<0,05)$, dentro das categorias de classificação de padrão racial, sendo mensurados valores superiores no outono. Não se observaram diferenças em relação aos machos $(\mathrm{p}>0,05)$.

A maior liquidez e disputa por fêmeas no período de outono, no que se refere às características de estrutura corporal e padrão racial, possivelmente esteve atrelada aos pesos destas nos distintos períodos. Visto que, fêmeas comercializadas na primavera foram mais pesadas frente as de outono $(\mathrm{p}<0,05)$, com, respectivamente, 210,62 e $157,14 \mathrm{~kg}$, para as médias gerias dos animais comercializados. Em relação aos machos, o peso de venda não diferiu nos distintos períodos $(\mathrm{p}>0,05)$, com pesos médios gerais de 166,44 e $167,08 \mathrm{~kg}$, na primavera e no outono, respectivamente. Assim, provavelmente, os resultados tenham ocorrido em função dos compradores se apegarem ao valor total do produto a ser adquirido (lote de animais), que, para animais maiores, ao se desembolsar o mesmo valor por unidade de peso vivo $(\mathrm{kg})$, envolve desembolsos mais elevados, resultando na possibilidade de compra de menor número de animais, para determinado montante. Dessa forma, as fêmeas de outono, por serem mais leves, receberam maiores valores por unidade de peso vivo.

Identificou-se superioridade de preços praticados por unidade de peso de fêmeas Brangus em relação às Braford e Devon ( $\mathrm{p}<0,05$ - Tabela 3$)$. Não houveram diferenças $(p>0,05)$ para os machos e para as demais raças de fêmeas.

Tabela 3. Percentuais de bezerros machos e fêmeas e valor por unidade de peso de acordo com a raça.

\begin{tabular}{|c|c|c|c|c|}
\hline \multirow{2}{*}{ Raça } & \multicolumn{2}{|c|}{ Machos } & \multicolumn{2}{|c|}{ Fêmeas } \\
\hline & $\%$ & $\mathbf{R} \$$ & $\%$ & $\mathbf{R} \$$ \\
\hline Aberdeen Angus & 27,27 & $5,22 \pm 0,26^{\mathrm{a}}$ & - & - \\
\hline Red Angus & 27,27 & $5,14 \pm 0,16^{\mathrm{a}}$ & 20,00 & $4,80 \pm 0,11^{\mathrm{ab}}$ \\
\hline Brangus & 6,06 & $5,04 \pm 0,06^{\mathrm{a}}$ & 13,33 & $5,22 \pm 0,05^{\mathrm{a}}$ \\
\hline Devon & 6,06 & $5,45 \pm 0,34^{\mathrm{a}}$ & 13,33 & $4,55 \pm 0,10^{\mathrm{b}}$ \\
\hline Hereford & 12,12 & $5,20 \pm 0,16^{\mathrm{a}}$ & 13,33 & $4,83 \pm 0,27^{\mathrm{ab}}$ \\
\hline Braford & 12,12 & $5,06 \pm 0,09^{\mathrm{a}}$ & 40,00 & $4,63 \pm 0,21^{b}$ \\
\hline Cruzas & 9,09 & $5,22 \pm 0,10^{\mathrm{a}}$ & - & - \\
\hline
\end{tabular}

*Valores com letras distintas na coluna diferem entre si pelo teste Tukey $(\mathrm{p}<0,05)$. 
Maior valorização por bezerros de raças sintéticas, onde se enquadra a raça Brangus, foi relatada por Christofari et al. (2008) ao analisarem a comercialização de bezerros durante o outono de 2004. No entanto, no presente estudo, a superioridade observada para fêmeas Brangus pode estar atrelada à reduzida oferta de animais desta raça nos leilões mensurados.

A bovinocultura de corte brasileira tem como característica a grande variabilidade de raças criadas, existindo registros de 22 raças europeias (Herd-Book Collares, 2018), 7 raças zebuínas (ABCZ, 2018) e inúmeras raças sintéticas oriundas de cruzamentos entre os grupos anteriores. No entanto, observou-se predomínio das raças Angus e Hereford, bem como de raças oriundas de cruzamentos e seleções dirigidas a partir destas. Os resultados obtidos ressaltam a importância que ambas as raças têm adquirido no Estado do Rio Grande do Sul durante os últimos anos, possivelmente alicerçadas nos programas de incentivo para a criação das mesmas, desenvolvidos pelas associações de seus criadores, com o intuito de fortalecer e integrar a cadeia produtiva da carne, em consonância com o pagamento de bonificações para animais abatidos conforme os padrões das mesmas (Vaz et al., 2002; ABA, 2018; ABHB, 2018; CNA, 2018).

$\mathrm{O}$ peso dos animais interferiu no preço de comercialização ( $\mathrm{p}<0,05$ - Tabela 4), onde fêmeas mais leves, de até $150 \mathrm{~kg}$, recebem maior valorização por quilograma de peso vivo comparadas às mais pesadas $(>180 \mathrm{~kg})$, e machos de pesos intermediários, entre 150 e $180 \mathrm{~kg}$, foram mais valorizados que os pesados $(>180 \mathrm{~kg})$.

Tabela 4. Número de lotes, valor por unidade de peso $(\mathrm{R} \$ / \mathrm{Kg})$ e número total de lotes não vendidos de bezerros machos e fêmeas de acordo com as faixas de peso.

\begin{tabular}{|c|c|c|c|}
\hline & \multicolumn{3}{|c|}{ Faixas de Peso (kg) } \\
\hline Variáveis & $\leq 150$ & $150-180$ & $>180$ \\
\hline \multicolumn{4}{|l|}{ Machos } \\
\hline $\mathrm{N}^{\mathrm{o}}$ Lotes & 18 & 22 & 17 \\
\hline $\mathrm{R} \$ / \mathrm{Kg}$ & $5,16 \pm 0,18^{\mathrm{ab}}$ & $5,28 \pm 0,23^{\mathrm{a}}$ & $5,05 \pm 0,12^{b}$ \\
\hline Lotes não vendidos & - & 1 & 2 \\
\hline \multicolumn{4}{|l|}{ Fêmeas } \\
\hline $\mathrm{N}^{\mathrm{o}}$ Lotes & 5 & 2 & 8 \\
\hline $\mathrm{R} \$ / \mathrm{Kg}$ & $4,96 \pm 0,28^{\mathrm{a}}$ & $4,87 \pm 0,07^{\mathrm{ab}}$ & $4,60 \pm 0,17^{b}$ \\
\hline Lotes não vendidos & - & - & - \\
\hline
\end{tabular}

*Valores com letras distintas na linha diferem entre si pelo teste Tukey $(\mathrm{p}<0,05)$.

Estes resultados, provavelmente estejam envolvidos ao valor total dos produtos, que para animais menores é mais reduzido. Outra questão pode estar relacionada ao ganho de peso compensatório, pois, como os bezerros apresentam idades semelhantes no momento da comercialização, possivelmente os mais leves tenham sido submetidos a condições nutricionais mais restritas, tendo a possibilidade de apresentarem ganhos de pesos superiores durante a fase de recria (Trindade Júnior et al., 2015).

O tempo de permanência em pista não foi afetado por nenhuma das características estudadas ( $p>0,05$ - dados não apresentados). Vale destacar que a proporção de machos e fêmeas ofertados foi bem distinta nas diferentes épocas do ano, com crescimento considerável na presença de fêmeas no período de primavera ( $47 \%$ frente a $21 \%$ no outono). Fato este, que pode ser justificado pelo retorno de aquisição de bezerros machos para exportação durante o período, o que reduziu a oferta de animais deste sexo na grande maioria de leilões da região do estudo. De qualquer forma, em ambos os períodos, houve menor oferta de bezerras, o que possivelmente esteja relacionado à retenção de fêmeas para reposição, visto que as probabilidades de nascimentos esperados para as estações reprodutivas são de $50 \%$ para ambos os sexos.

Quanto aos preços praticados em relação ao sexo dos animais, obteve-se $\mathrm{R} \$ 5,17$ e $\mathrm{R} \$ 4,76$, para machos e fêmeas, respectivamente. Resultado que corrobora os achados de Smith et al. (2000), Crespo et al. (2006), Troxel e Barham (2007), Koetz Junior et al. (2014) e Fornari et al. (2016) estudando leilões de bezerros realizados ao redor do mundo, de que o preço médio dos machos é superior ao das fêmeas. O preço praticado pelos machos foi $8,61 \%$ maior que o das fêmeas. Índice semelhante foi relatado por Fornari et al. (2016), de $7,8 \%$ de superioridade dos machos, estudando a 
comercialização de bezerros no Estado de Santa Catarina, Brasil.

\section{Conclusão}

Conclui-se que lotes de animais mais uniformes quanto às características raciais recebem maior valorização no momento de comercialização. As faixas de peso interferem no preço de venda, sendo os animais mais pesados, com peso superior a $180 \mathrm{~kg}$, os menos valorizados. O período de comercialização e a raça dos animais interferem no preço por unidade de peso das fêmeas.

\section{Conflito de Interesse}

Os autores declaram não haver conflito de interesses.

\section{Comissão de Ética}

As observações não causaram nenhuma intervenção nos animais, além do manejo básico efetuado na rotina dos leilões, desta forma o projeto não foi submetido ao comitê de ética no uso de animais (CEUA).

\section{Referências}

ABA - Associação Brasileira de Angus. Carne Angus. 2018. Disponível em: $<$ http://angus.org.br/carne-angus-2/>. Acesso em: 01 abr. 2018.

ABCZ - Associação Brasileira de Criadores de Zebú. Raças zebuínas. 2018. Disponível em: <http://www.abcz.org.br/Home/Conteudo/23 985-Racas-Zebuinas>. Acesso em: $01 \mathrm{abr}$. 2018.

ABHB - Associação Brasileira de Hereford e Braford. Carne Certificada Hereford. 2018. Disponível em: <http://www.carnehereford.com.br/historico/ o-programa/>. Acesso em: 01 abr. 2018.

Ayres, M.; Junior, M.A.; Ayres, D.L.; Santos, A.A.S. BioEstat 5.3: Aplicações estatísticas nas áreas das Ciências Biomédicas. Belém: Sociedade Civil Mamirauá, 2007. 324p.

CNA - Confederação da Agricultura e Pecuária do Brasil. Certificação de Raças Bovinas. 2018. Disponível em: <http://www.cnabrasil.org.br/carnebonificada>. Acesso em: 11 abr. 2018.

Christofari, L.F.; Barcellos, J.O.J.; Braccini Neto, J.; Oaigen, R.P.; Canozzi, M.E.A.; Wilbert, C.A. Manejo da comercialização em leilões e seus efeitos no preço de bezerros de corte.
Revista Brasileira de Zootecnia, 38(1): 196203, 2009.

Christofari, L.F.; Barcellos, J.O.J.; Braccini Neto, J.; Oaigen, R.P.; Santos, A.P.; Canozzi, M.E.A. Efeitos do peso vivo sobre a comercialização de bezerros de corte em leilões. Arquivo Brasileiro de Medicina Veterinária e Zootecnia, 62(2): 419-428, 2010.

Christofari, L.F.; Barcellos, J.O.B.; Costa, E.C.; Oaigen, R.P.; Neto, J.B; Grecellé, R.A. Tendências na comercialização de bezerros no Rio Grande do Sul relacionadas às características genéticas. Revista Brasileira de Zootecnia, 37(1): 171-176, 2008.

Crespo, B.L.; Patisson, C.O.; Innella, A.B. Variabilidad de corto plazo en la formación de precios en el mercado vacuno de reposición. Série técnica no 155 . INIA, Montevideo, 2006. $70 \mathrm{p}$.

Euclides Filho, K.; Euclides, V.P.B. Desenvolvimento recente da pecuária de corte brasileira e suas perspectivas. In: Pires, A.V. Bovinocultura de corte. Piracicaba: Fealq, 2010. p.11-40.

Fornari, G.B.; Menegassi, S.R.O.; Pereira, G.R.; Oliveira, T.E.; Barcellos, J.O.J. Factors affecting the selling prices of calves in auctions in Santa Catarina State, Brazil. Revista Brasileira de Zootecnia, 45(10): 632-638, 2016.

Herd-Book Collares. Raças. 2018. Disponível em: <http://www.herdbook.org.br/institucional/in dex.asp?pag=conteudo/cabracbov.asp $>$. Acesso em: 03 abr. 2018.

Koetz Junior, C.; Lopes, F.G.; Barca Júnior, F.A.; Claus, L.A.M.; Canozzi, M.E.A.; Menegassi, S.R.O.; Dill, M.D.; Barcellos, J.O.J. Phenotypic factors and genetic group on the selling price formation of beef calves sold in auctions at the Northern region of Paraná State, Brazil. Ciência Rural, 44(4): 752-756, 2014.

Koury Filho, W.; De Albuquerque, L.G.; De Alencar, M.M.; Forni, S.; Silva, J.A.I.V.; Lôbo, R.B. Estimativas de herdabilidade e correlações para escores visuais, peso e altura ao sobreano em rebanhos da raça Nelore. Revista Brasileira de Zootecnia, 38(12): 2362-2367, 2009.

Machado Filho, C.A.P. Leilões de animais no Brasil. Revista de Administração, 29(1): 7682, 1994. 
Oiagen, R.P.; Barcellos, J.O.J.; Christofari, L.F.; Neto, J.B.; Oliveira, T.E.; Prates, E.R. Análise da sensibilidade da metodologia dos centros de custos mediante a introdução de tecnologias em um sistema de produção de cria. Revista Brasileira de Zootecnia, 38(6): 1155-1162, 2009.

Sachs, R.C.C.; Martins, S.S. Análise do Comportamento dos Preços do Boi Gordo e do Bezerro na Pecuária de Corte Paulista, Janeiro de 1995 a Abril de 2006: Uma Aplicação do Modelo VAR. Revista de Economia Agrícola, 54(1): 75-85, 2007.

Schoroeder, T.; Mintert, J.; Brazle, F.; Grunewald, O. Factors Affecting Feeder cattle Price Differentials. Western Journal of Agricultural Economics, 13(1): 71-81, 1988. Smith, S.C.; Gill, D.R.; Jones, S.C.; Gardner, B.A. Effect of selected characteristics on the sale price of feeder cattle in eastern Oklahoma. Stillwater, OK: Oklahoma Cooperative Extension Service, Division of Agricultural Science and Natural Resources, Oklahoma State University. Report ${ }^{\circ} \mathrm{E}-955,2000.7 \mathrm{p}$.
Spitzer, J.C. Influences of nutrition on reproduction in beef cattle. In: Morrow, D.A. (Ed.). Current therapy in Theriogenology. 2. ed. Philadelphia: W.B. Saunders, 1986. p. 231-234.

Trindade Júnior, G.; Silva, R.R.; Carvalho, G.G.P.; Silva, F.F.; Negrão, J.A.; Barroso, D.S.; Dias, D.L.S.; Costa, P.B. Ganho compensatório de novilhas mestiças suplementadas em pastagens sob avaliação do perfil hormonal e parâmetros sanguíneos. Semina: Ciências Agrárias, 36(3): 1481-1494, 2015.

Troxel, T.R.; Barham, B.L. Comparing the 2000 and 2005 factors affecting the selling price of feeder cattle sold at Arkansas livestock auctions. Journal of Animal Science, 85(12): 3425-3433, 2007.

Vaz, F.N.; Restle, J.; Pacheco, P.S.; Freitas, A.D.; Peixoto, L.D.O.; Carrilho, C.D.O. Características de carcaça e da carne de novilhos superprecoces de três grupos genéticos, gerados por fêmeas de dois anos. Revista Brasileira de Zootecnia, 31(5): 1973-1982, 2002. 